\title{
Comparative study of electron conduction in azulene and naphthalene
}

\author{
SUDIPTA DUTTA, S LAKSHMI and SWAPAN K PATI* \\ DST Unit on Nanoscience and Theoretical Sciences Unit, Jawaharlal Nehru Centre for Advanced Scientific \\ Research, Bangalore 560 064, India
}

\begin{abstract}
We have studied the feasibility of electron conduction in azulene molecule and compared with that in its isomer naphthalene. We have used non-equilibrium Green's function formalism to measure the current in our systems as a response of the external electric field. Parallely we have performed the Gaussian calculations with electric field in the same bias window to observe the impact of external bias on the wave functions of the systems. We have found that the conduction of azulene is higher than that of naphthalene inspite of its intrinsic donor-acceptor property, which leads a system to more insulating state. Due to stabilization through charge transfer the azulene system can be fabricated as a very effective molecular wire. Our calculations show the possibility of huge device application of azulene in nano-scale instruments.
\end{abstract}

Keywords. Azulene; naphthalene; NEGF; molecular wire.

\section{Introduction}

Electron transport through a single molecule has generated a great deal of interest both theoretically and experimentally in recent times because of its wide variety of device applications (Ratner et al 1994, 1996; Dorogi et al 1995; Joachim et al 1995; Joachim and Roth 1997; Jortner and Ratner 1997; Zhou et al 1997; Alivisatos et al 1998; Zahid et al 2003). These types of molecules are believed to be the potential components of future nanoscale computational or electronic devices. Recent advances in experimental techniques have allowed fabrication and measurement of current through such nanoscale systems. Various molecules have already been demonstrated to behave as wires, switches, diodes, RAMs etc (Reed et al 1997, 2001; Cui et al 2001; Kushmerick et al 2004). In most of the experiments, the nanoscale material is an organic molecule or a $\pi$-conjugated polymer. Many theories too have been developed from empirical to semi-empirical and $a b$ initio level to describe the electrical response functions of the nanomaterials (Lakshmi and Pati 2004, 2005; Lakshmi et al 2005; Sengupta et al 2006; Dutta et al 2007; Dutta and Pati 2008a, b).

The ability of a molecule to switch between off and on states is one of the most important applications of these nanoscale materials. Experimentally, this has been observed in many organic molecules with various donor and acceptor substituents. Such an unusual and interesting behaviour of organic molecules with donor and acceptor moieties stimulates us to study the electronic conduction in azulene molecule and to compare that with its isomer, naphthalene. Although naphthalene and azulene are structurally similar

*Author for correspondence (pati@jncasr.ac.in) with the same number of carbon and hydrogen atoms and the same number (ten) of $\pi$ electrons, the properties of the azulene molecule differ from that of its isomer in many respects (Streitwieser 1961; Salem 1966; Bergman and Pullman 1971; Vogtl 1989). The geometry of the azulene molecule consists of fused five and seven membered rings which contribute to the intense blue colour and the large dipole moment $(\mu=0.8-1.08 D)$ for a hydrocarbon system (Anderson and Steckler 1959; Tobler et al 1965). It has interesting photophysical properties like $S_{2} \rightarrow S_{0}$ fluorescence (Beer and Longuet-Higgins 1955), large hyperpolarizability (Morley 1989) etc. The observed dipole moment is due to the partial charge transfer from the seven membered ring to the five membered ring which gives the molecule aromatic stability obeying Huckel $4 n+2$ rule in both the rings with $n=1$ and this gives the molecule intense blue colour. Therefore, the ground state consists of a donor seven membered ring and an acceptor five membered ring fused together. On the contrary, naphthalene molecule consists of two fused six membered rings with six electrons in each ring. Here, there is no occurrence of charge transfer to gain aromatic stability which ensures the zero dipole moment and thus making it appear colourless.

Due to the interesting charge transfer properties of the two thiolated molecules, we have studied their $I-V$ characteristics between two gold electrodes using the nonequilibrium Green's function methods, already widely reported in the literature. We apply the electric field along the principle $C_{2}$ axis in both the directions. Parallely, we use quantum chemical methods to study the effect of electric field on their molecular orbitals that are accessed during the transport.

In $\S 2$, we have discussed about our computational details. $\S \S 3$ and 4 contain the results with discussion and conclusion of our work, respectively. 


\section{Computational method}

Firstly, to study the effect of electric field on the frontier molecular orbitals of both the molecules, we optimized the ground state structures of azulene and naphthalene with two thiol groups placed at the furthest positions as shown in figure 1 using the density functional methods with 6$31++G(d)$ basis set and density functional exchange correlation, $B 3 L Y P$. We then optimize the structures of both the molecules using the same basis set in the presence of electric field, applied along the chain axis of the molecules. We vary the electric field in the interval of 0.001 a.u. and 1 a.u. $=5.142 \times 1011 \mathrm{~V} / \mathrm{m}$. Vibrational analysis is then performed on each optimized structure to check whether, what we obtain are the stable minimum energy structures with all real frequencies. Then we calculate the Fock and overlap matrices for the ground state molecules which we use later for the calculation of current using non-equilibrium Green's function (NEGF) method (Datta 1995). All calculations are performed with the standard Gaussian-03 (Frisch et al 2003) package.

Parallely, we study the equilibrium properties of the molecules before connecting them with the electrodes. We first calculate the Green's function, $G$, in equilibrium situation using the formula,

$$
G=\frac{1}{E S-H+i \eta},
$$

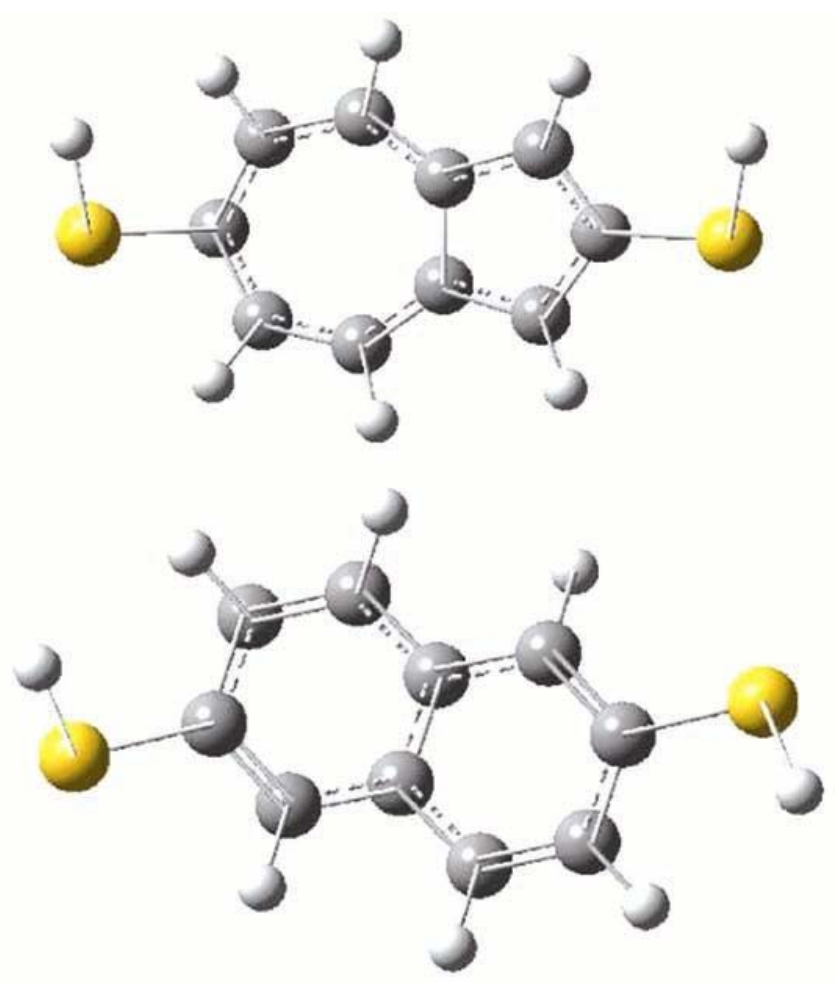

Figure 1. Structure of azulene and naphthalene with two thiol groups linked at furthest positions. where $E$ is the electron injection energy, $S$ and $H$ are the overlap and Fock matrices, respectively. $\eta$, a small arbitrary broadening parameter, arises mostly due to temperature. We obtain $S$ and $H$ from the density functional calculations with Gaussian-03 package. The hydrogens attached to the thiol groups are removed by modifying the Hamiltonian and overlap matrices at those positions and these are then used for the calculation of the Green's function. From the eigen energies of the relaxed system, the Fermi energy, $E_{\mathrm{F}}$, of the electrodes is calculated assuming that it falls midway between the equilibrium highest-occupied molecular orbital (HOMO) and lowestunoccupied molecular orbital (LUMO) energies of the molecule alone. The Fermi energy can be anywhere between the HOMO and LUMO and is generally considered to be a fitting parameter. Then we calculate the density of states and the transmission $(T(E))$ between the terminal sulfur atoms $\left(S_{1}\right.$ and $\left.S_{2}\right)$.

$$
T(E) \propto\left|G_{S_{1} S_{2}}\right|^{2} \eta^{2} \text {. }
$$

The external electric field applied on the system has the form of a ramp potential, distributed over all the sites in such a way that the potential, $V_{\mathrm{i}}$, at site $i$ becomes

$$
-\frac{V}{2}+V \frac{x(i)-x\left(S_{1}\right)}{x\left(S_{2}\right)-x\left(S_{1}\right)}
$$

where $V$ is the applied voltage and $x(i), x\left(S_{1}\right)$ and $x\left(S_{2}\right)$ are the $x$-coordinates of $i$ th atom, 1 st and 2 nd sulfur atoms, respectively. This form of the potential ensures that the bias varies from $-V / 2$ to $V / 2$ across the molecule. The potential adds an extra diagonal term $\sum_{i} V_{i} a_{i}^{\dagger} a_{i}$ to the Fock matrix.

The effect of electrodes on the molecule has been incorporated through the well known self-energy term, $\Sigma$, for gold electrodes. With this imaginary self-energy terms the Hamiltonian gets modified as

$$
H=H_{\mathrm{i}}+\sum_{\mathrm{L}}+\sum_{\mathrm{R}},
$$

where $H_{\mathrm{i}}$ is the initial Hamiltonian for only the molecule. $\Sigma_{\mathrm{L}}$ and $\sum_{\mathrm{R}}$ are self-energies of left and right electrodes, respectively. Having obtained the modified Hamiltonian, the current through the molecule is obtained using Landauer's $(1957,1981)$ formula,

$$
I(V)=\frac{2 e}{h} \int_{-\infty}^{\infty} \mathrm{d} E\left[\operatorname{Tr}\left(\Gamma_{\mathrm{L}} G \Gamma_{\mathrm{R}} G^{\dagger}\right)\right],
$$

where $\Gamma_{L}$ and $\Gamma_{R}$ are the anti-Hermitian parts of the selfenergy matrices, $\Sigma_{\mathrm{L}}$ and $\sum_{\mathrm{R}}$, respectively

$$
\Gamma_{\mathrm{L}, \mathrm{R}}=i\left(\sum_{\mathrm{L}, \mathrm{R}}-\sum_{\mathrm{L}, \mathrm{R}}^{\dagger}\right),
$$

which describe the broadening of the energy levels due to the coupling of the molecule to the electrodes. The Green's function is obtained as the inverse of the modified Hamiltonian 


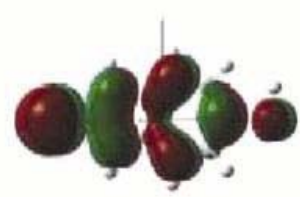

I

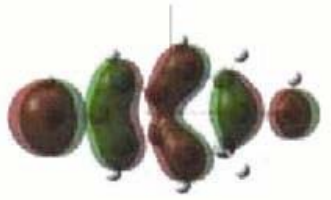

II

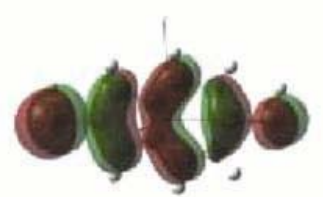

III

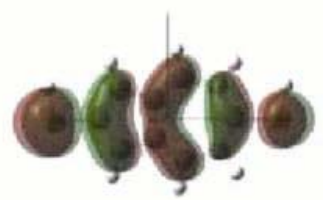

IV

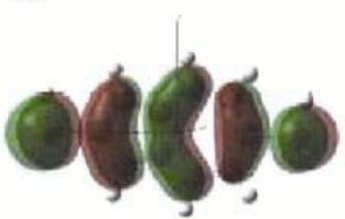

$\mathrm{V}$

Figure 2. HOMO plots for the electric fields, 0.01 (I), 0.008 (II), 0.004 (III), 0.002 (IV), 0 (V), -0.002 (VI), -0.004 (VII), -0.008 (VIII) and -0.01 (IX) for the azulene molecule with two terminal

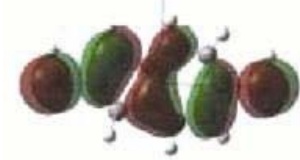

VI thiol groups (unit of electric field is a.u).

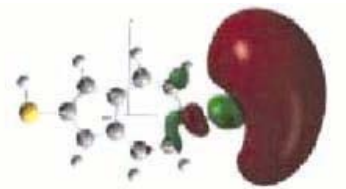

I

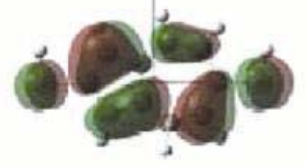

VII

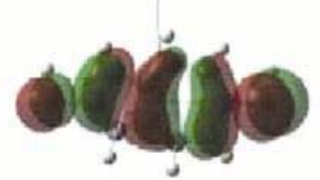

VIII

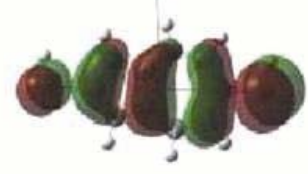

IX

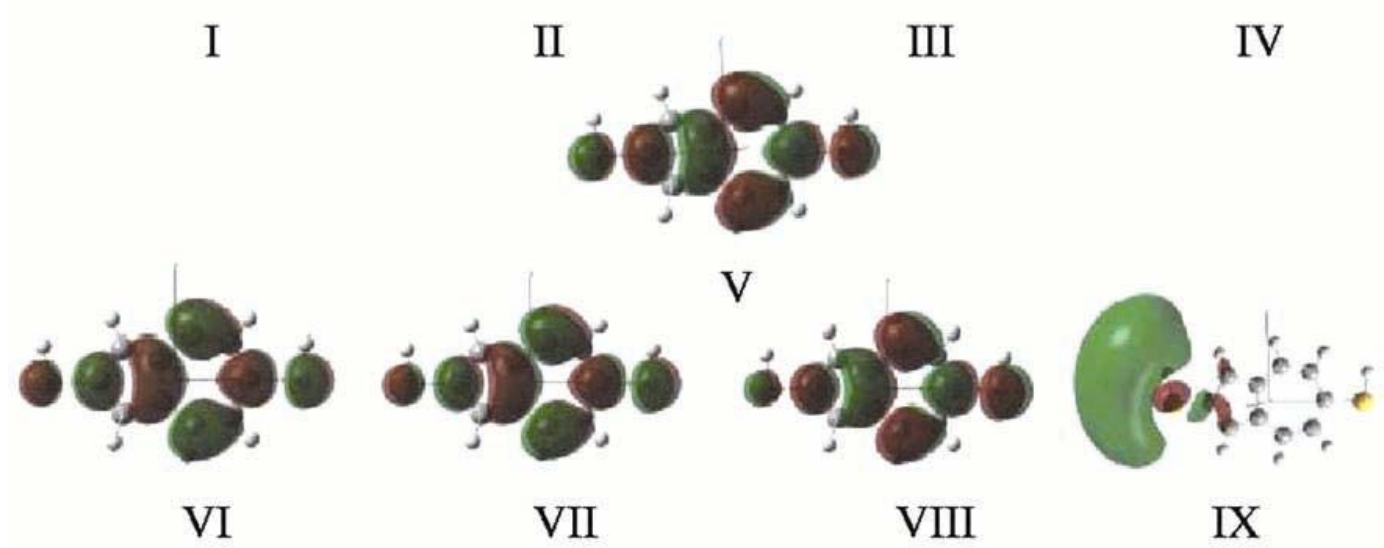

Figure 3. LUMO plots for the electric fields 0.01 (I), 0.008 (II), 0.004 (III), 0.002 (IV), 0 (V), $-0 \cdot 002$ (VI), -0.004 (VII), -0.008 (VIII) and -0.01 (IX) for the azulene molecule with two terminal thiol groups (unit of electric field is a.u).

$$
G=\frac{1}{E S-\left[H+i\left(\Sigma_{\mathrm{L}}+\Sigma_{\mathrm{R}}\right)\right]} .
$$

Using this Green's function we calculate the current. We calculate the transmission using the formula

$$
T(E)=\operatorname{Tr}\left(\Gamma_{\mathrm{L}} G \Gamma_{\mathrm{R}} G^{\dagger}\right) .
$$
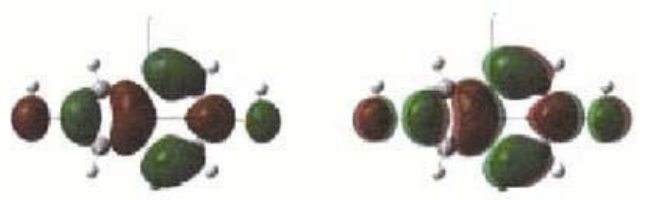

IV

\section{Results and discussion}

On application of electric field, the HOMO and LUMO of azulene changes from the ground state as can be seen from figures 2 and 3, respectively. These figures clearly show that the effect of electric field, applied along posi- 
tive $x$-axis differs a lot from that along opposite direction. In case of HOMO, the electron density is delocalized over the whole molecule and it shows electron density shift along the bias direction. LUMO electron density is also delocalized but at higher bias value it shows complete localization of electron density at one end which depends on the bias direction. The ground state dipole moment also changes differently for the electric field in either directions. From figure 4 it can be seen that for both the electric field directions, the dipole moment of azulene system increases. In absence of electric field the azulene molecule has some dipole moment which increases with increasing bias in positive $x$-axis direction. But the electric field along negative $x$-axis first reduces the dipole moment followed by a monotonic increase which can be seen clearly from the inset of figure 4 . This observation is in agreement with the fact that the electron flows from the seven membered ring to the five membered ring. The

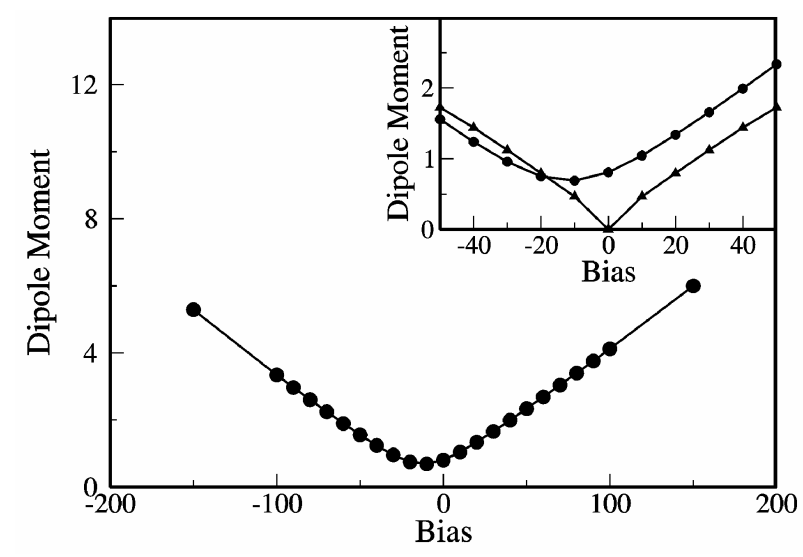

Figure 4. Variation of dipole moment of the azulene system as a function of electric field. Inset shows the dipole moment for both azulene (circle) and naphthalene (triangle) in a smaller bias window.

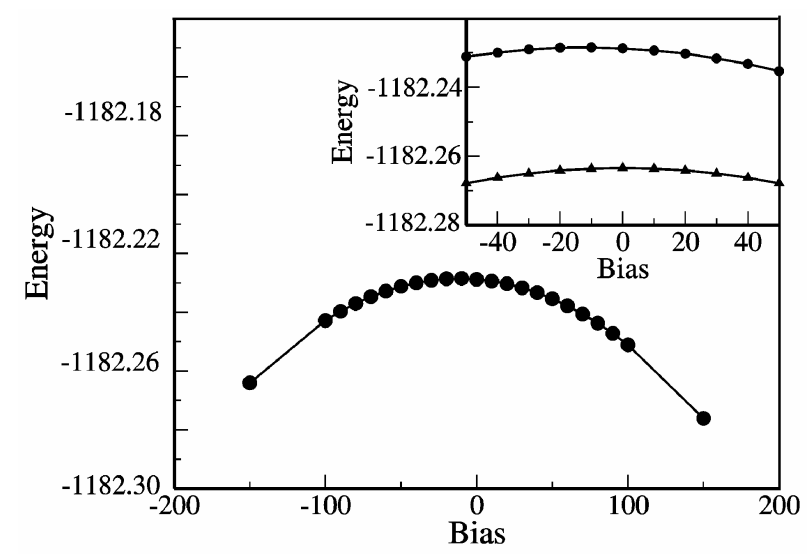

Figure 5. Energy of the azulene system as a function of external electric field. Inset shows energy for both azulene (circle) and naphthalene (triangle) in a smaller bias window. electric field in the opposite direction forces the electrons to go from five membered ring to seven membered ring and thus the dipole moment reduces from the ground state dipole moment initially for lower bias. Further increase in bias in this direction accumulates the charge on the seven membered ring resulting in a dipole moment increase. But the dipole moment of naphthalene molecule increases with increasing bias in both the directions from the ground state zero dipole moment.

Figure 5 shows the variation of the energy of azulene system as a function of external electric field. The energy decreases with increasing electric field in $+v e x$-axis, since the system gets stabilized for the charge transfer on application of electric field in this direction. But field along -ve $x$-axis initially destabilizes the molecule at smaller bias values followed by a monotonic decrease in energy with increasing bias. The initial destabilization is attributed to the fact that, the small bias tries to force the

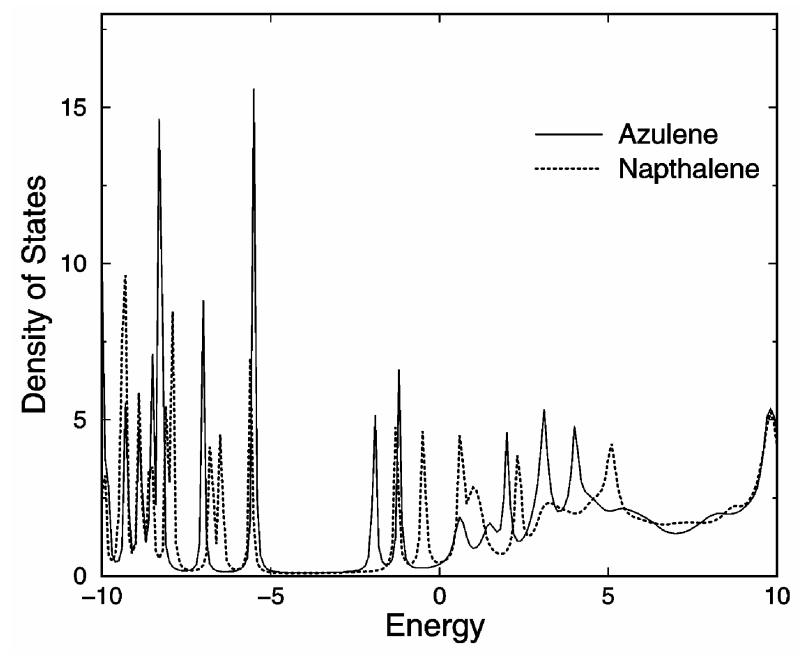

Figure 6. Density of states as a function of energy for azulene and naphthalene molecules with two-terminal thiol group.

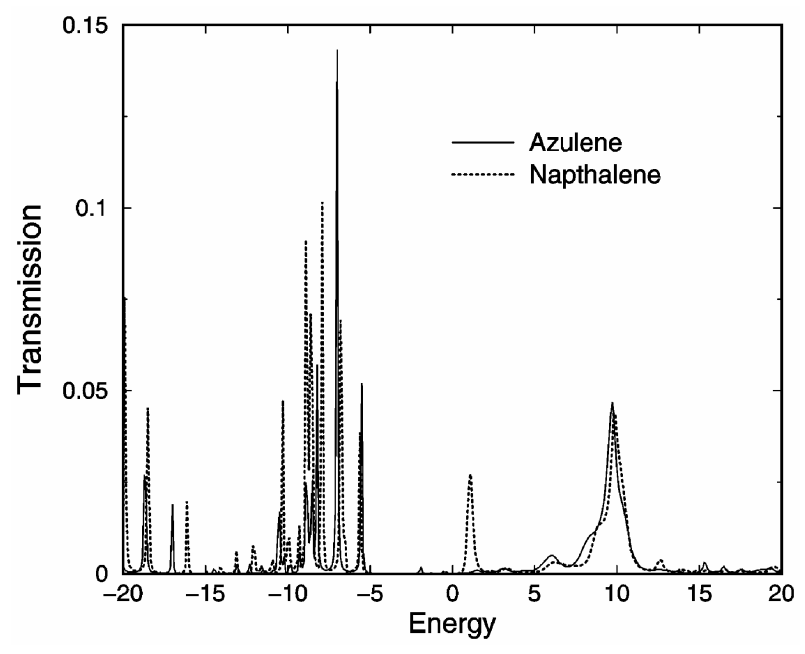

Figure 7. Transmission as a function of energy for azulene and naphthalene molecules with two-terminal thiol group. 
electrons to flow in its direction in which the system loses its aromatic stability. But for larger bias values, the system again behaves like a charge transfer compound and starts getting stabilized. Inset of figure 5 shows the comparison of the energy variation of both azulene and naphthalene systems as a function of bias. The energy of naphthalene molecule decreases from the ground state energy on application of external bias, applied in both the directions.

So, it is clear from the above discussion that the electron density rearranges differently for electric fields along -ve and +ve $x$-axes in case of azulene system, whereas in case of naphthalene system the effects of electric field along both the directions are same due to its symmetric structure. Now, we calculate the current as a response of applied electric field using the NEGF formalism for both azulene and naphthalene systems. First, we plot the equilibrium, i.e. the zero bias density of states (DOS) in figure 6 and transmission in figure 7. From the DOS plot it can be clearly seen that the HOMO-LUMO gap in case of naphthalene $(\sim 4 \mathrm{eV})$ is higher than that of azulene $(\sim 3 \mathrm{eV})$ system. HOMO energy for naphthalene is lower than that of azulene and as a result, naphthalene is stabler than its isomer. From the plot of equilibrium transmission, it is very evident that the HOMO of both azulene and naphthalene conduct well, whereas the LUMO of both show negligible transmission. Hence, in our current calculations, we place the Fermi energy closer to the HOMO to probe conduction through the occupied levels.

To get proper molecular understanding, we keep the bias window more or less same for both types of calculations (NEGF and geometry optimization with electric field). In our calculations, the length scale of the molecules are 8-9 $\AA$, which ensures the bias window of Gaussian calculations similar to the NEGF calculations. Figure 8 shows the $I-V$ characteristics with steps for both azulene and naphthalene

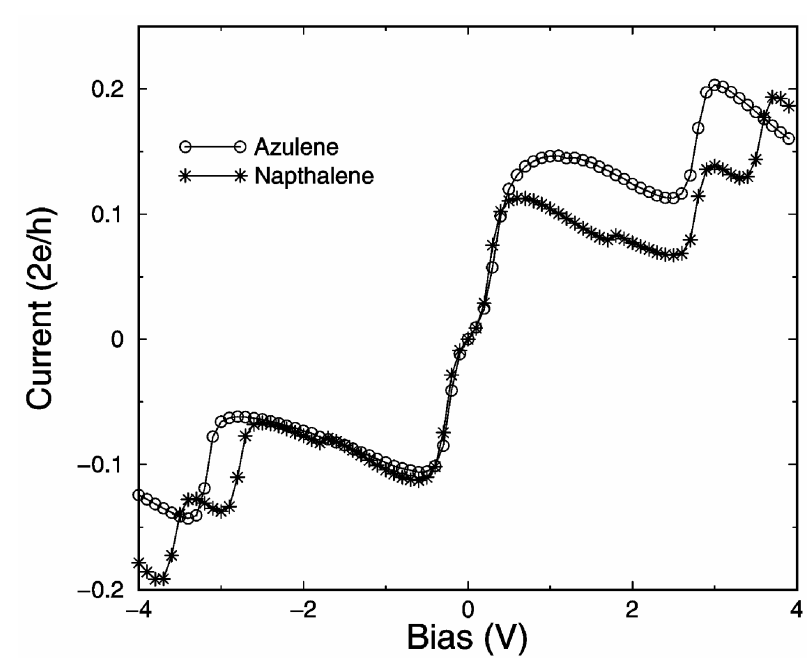

Figure 8. Current as a function of bias for azulene and naphthalene molecules with two-terminal thiol group. systems. The $I-V$ characteristics of naphthalene is completely symmetric in nature for both the bias directions, whereas for azulene it is not symmetric. It is very evident that, both the molecules show step-like behaviour in $I-V$ characteristics. These are the well known eigen-value staircases that appear whenever the electrochemical potential of the electrodes come in resonance with the molecular levels (Ratner 1994, 1996). We find that, in lower bias regime, both of them show nearly same current. Beyond a certain bias value along positive $x$-axis the azulene system shows more current than the naphthalene system. But for reverse bias the current of azulene system is lower than the naphthalene. It is due to the fact that, bias along +ve direction drives the electrons easily from seven membered ring to the five membered ring, whereas reverse bias drives the electrons opposite to the dipole moment direction. As a consequence, the current becomes lower than that of naphthalene which has symmetric electron cloud distribution over the two six-membered rings. At higher bias values the current shows a considerable decrease with increase in bias due to voltage induced localization. So, the interesting result, coming out of our calculations is that, the azulene molecule which has intrinsic donoracceptor property, shows more current than naphthalene; though it is expected to show more insulating behaviour.

\section{Conclusions}

We have performed both NEGF and Gaussian calculations for both azulene and naphthalene systems to measure the current passing through these systems on application of external electric field. We have observed the electron density rearrangement in both the systems in presence of bias. In case of azulene, the electrons flow from seven membered ring to the five membered ring more easily resulting in more stabilization with increase in bias. But for reverse bias, initially the dipole moment decreases with destabilization of the system due to electron flow in opposite direction. Our NEGF calculations show higher conductivity in azulene than that in naphthalene system. Our results reveal the possibility of potential device application of azulene as molecular wire in future nanoscale instruments.

\section{Acknowledgements}

One of the authors (SKP) thanks DST and CSIR, Government of India, for funding and (SD) acknowledges CSIR, Government of India, for a research fellowship.

\section{References}

Alivisatos A P et al 1998 Adv. Mater. (Germany) 101297

Anderson A G and Steckler B M 1959 J. Am. Chem. Soc. 81 4941 
Beer M B and Longuet-Higgins 1955 J. Chem. Phys. 231390

Bergman E D and Pullman B 1971 Aromaticity, pseudoaromaticity and anti-aromaticity (New York: Academic Press)

Cui X D et al 2001 Science 294571

Datta Supriyo 1995 Electronic transport in mesoscopic systems (Cambridge Studies in Semiconductor Physics and Microelectronic Engineering)

Dorogi, Gomez J, Andres R P and Reifenberger R 1995 Phys. Rev. B52 9071

Dutta S and Pati S K 2008a J. Phys.: Condens. Matter 20 075226

Dutta S and Pati S K 2008b J. Phys. Chem. B112 1333

Dutta S, Lakshmi S and Pati S K 2007 J. Phys.: Condens. Matter 19322201

Frisch M J et al 2003 Gaussian 03 (Pittsburgh, PA: Gaussian Inc.)

Joachim C and Roth S 1997 Atomic and molecular wires (Dordrecht: Kluwer Academic)

Joachim C, Gimzewski J K, Schlitter R R and Chavy C 1995 Phys. Rev. Lett. $\mathbf{7 4} 2102$

Jortner J and Ratner M A 1997 Molecular electronics (London: Blackwell)

Kushmerick J G et al 2002 Phys. Rev. Lett. 89086802

Lakshmi S and Pati S K 2004 J. Chem. Phys. 12111998
Lakshmi S and Pati S K 2005 Phys. Rev. B72 193410

Lakshmi S, Datta A and Pati S K 2005 Phys. Rev. B72 045131

Landauer R 1957 IBM J. Res. Dev. 1223

Landauer R 1981 Phys. Lett. A85 91

Morley J O 1989 J. Chem. Soc. Perkin Trans. II 103

Ratner M A et al 1994 J. Chem. Phys. 101 5173, 6849, 6856

Ratner M A et al 1996 J. Chem. Phys. 1047296

Reed M A et al 1997 Science 278252

Reed M A et al 2001 Appl. Phys. Lett. 783735

Salem L 1966 The molecular orbital theory for conjugated systems (New York: Benjamin)

Sengupta S, Lakshmi S and Pati S K 2006 J. Phys.: Condens. Matter 189189

Streitwieser A Jr 1961 Molecular orbital theory for organic chemists (New York: Wiley)

Tobler H J, Bauder A and Gunthard Hs H 1965 J. Mol. Spectrosc. 18239

Vogtle F 1989 Reizvolle Molekule der organischen chemie (Stuttgart: Teubner)

Zahid F, Paulsson M and Datta S 2003 Advanced semiconductors and organic nano-techniques (ed.) H Morkoc (New York: Academic Press)

Zhou C, Deshpande M R and Reed M A 1997 Appl. Phys. Lett. $\mathbf{7 1} 611$ 